\title{
Is South Africa ready for the future of human germline genome editing? Comparing South African law and recent proposals for global governance
}

\author{
T Kamwendo, ${ }^{1}$ LLM; B Shozi, ${ }^{2,3}$ LLM, PhD \\ 1 Private Law Department, Faculty of Law, University of the Free State, Bloemfontein, South Africa \\ ${ }^{2}$ Institute for Practical Ethics, Division of Arts and Humanities, University of California, San Diego, USA \\ ${ }^{3}$ School of Law, College of Law and Management Studies, University of KwaZulu-Natal, Durban, South Africa
}

Corresponding author: TKamwendo (Kamwendot@ufs.ac.za)

\begin{abstract}
Over the past few years, developments in the science of precise editing of human genomes using CRISPR-Cas9 have led many countries that lack specific laws in this area, such as South Africa (SA), to contemplate legal reform. Thaldar et al. recently published five principles to guide legal reform in SA on heritable genome editing. In a similar vein, concerns about the global impact of human germline genome editing have led to calls for a global regulatory mechanism. This is what the World Health Organization has tried to achieve with the recently published 'Draft governance framework for human genome editing'. In this article, we compare the policies proposed by the draft framework to the current SA legal position, as well as the five guiding principles. The article concludes that SA law is in need of reform in order to meet the global standards that the draft framework seems to be moving towards.
\end{abstract}

S Afr J Bioethics Law 2021;14(3):97-100. https://doi.org/10.7196/SAJBL.2021.v14i3.761

\begin{abstract}
Advances in genome editing technologies such as CRISPR-Cas9 have offered immense hope for humanity due to their ability to ameliorate or prevent certain human conditions and diseases. However, while the potential relief from these diseases is embraced by many, there is still considerable public disquiet with regard to genome editing, particularly the modification of the human genome in the germline. These concerns range from the belief that human involvement in our own evolution is unethical, to unknown effects of genome editing on the individual and society at large and the fear of its potential abuse. ${ }^{[1]}$ CRISPR-Cas9's extraordinary potential, and the possibility of its abuse, illuminate the need for a comprehensive regulatory framework designed to promote scientific discovery while striking a balance with ethical boundaries. This has left several states that lack explicit regulation, including South Africa (SA), questioning how they should regulate the use of this technology in the face of the ongoing controversy about the technology's moral status and the extent of its possible applications. In this article, we consider what the policy in SA ought to be in light of recent developments in the discourse about global regulation of human germline genome editing.

In 2020, the World Health Organization (WHO) expert advisory committee, after due consultation with scientists, academics and other stakeholders, established the 'Draft governance framework for human genome editing.[2] This framework provides insight into what global regulation on human germline genome editing may be, and in this article, we compare the recommendations of the WHO advisory committee against SA's current legal framework. Recognising that SA policy on human germline genome editing is currently unclear and in need of reform, ${ }^{[3]}$ we will analyse the draft framework through
\end{abstract}

the lens of the five principles proposed by Thaldar et al. ${ }^{[4]}$ These principles assist in providing systematic guidance on what SA policy formation on human germline genome editing should entail in light of prevailing ethical principles, legal norms and human rights.

This article begins with a discussion on the current proposals for the global regulation of genome editing by giving an overview of the draft framework and its founding principles. It then compares the policies proposed in the draft framework with the current SA legal position in an attempt to align the SA policy on germline genome editing with global standards, while also adhering to SA norms and values. This article therefore aims to stimulate debate by encouraging policy-makers to critically assess the suitability of the draft framework in regulating the future of human germline genome editing.

\section{WHO draft governance framework on human genome editing: A general overview}

The WHO Advisory Committee on Developing Global Standards for Governance and Oversight of Human Genome Editing was tasked with developing appropriate global governance mechanisms for human genome editing. Accordingly, in 2020, after due consultation with relevant stakeholders, the committee released a 'Draft governance framework on human genome editing'. The global regulatory system as proposed in the draft framework is based on principles of promoting wellbeing, transparency, due care, responsible science, respect for persons and fairness. These principles demonstrate the overarching 
challenge of balancing the need to promote scientific advances against highly contentious socioethical concerns. Within the entire system of oversight, the draft framework acknowledges that different regulatory approaches for different applications of genome editing may be more suitable, taking into account the varying considerations across the sectors. ${ }^{[2]}$ Furthermore, since applications of genome editing are likely to transcend national boundaries, there is need for regulation at both national and transnational levels, particularly regarding highly contentious germline editing. This, in turn, leads to a wide range of responsibilities for SA in attempting to develop a good governance framework for germline editing.

\section{Principles for governance of human germline editing}

In this section, the article compares SA law on heritable genome editing with the WHO draft governance framework, using the five principles proposed by Thaldar et al. ${ }^{[4]}$ as guidance for how genome editing should be regulated in SA. This discussion will focus primarily on the regulation of the potential clinical applications of germline editing. In this regard, we will highlight how some of the proposed guidelines in the draft framework are disproportionate to the risk, and if implemented, would be tantamount to over-regulation, thus calling for an exceptionalist approach.

\section{Principle 1: Human germline editing should be regulated, not banned}

The SA position regarding the permissibility of human germline editing is somewhat unclear. As in many other countries across the globe, most laws relating to human germline genome editing were drafted in times of imprecise genome editing technologies. ${ }^{[5]}$ Section 57 of the National Health Act No. 61 of $2003(\mathrm{NHA})^{[6]}$ criminalises reproductive cloning of humans. Reproductive cloning has been defined as the manipulation of genetic material in order to achieve the reproduction of a human being. Thaldar et al. ${ }^{[4]}$ have argued that while human germline editing cannot be regarded as cloning per se, germline editing involves the manipulation of genetic material, and hence it may be construed that such activities fall within the scope of 'human reproductive cloning' as defined by the NHA, thereby rendering it illegal. Whereas a different interpretation can be inferred by applying principles of statutory interpretation, the absence of case law renders the position unclear. ${ }^{[4]}$ Then again, the Health Professions Council of South Africa (HPCSA) ${ }^{[7]}$ prohibits research relating to germline gene therapy but is silent on the clinical application of germline editing. And so, the major regulatory challenge that ought to be addressed is: should human germline editing be allowed in SA, and if so, under what conditions?

Although clinical applications of germline editing give rise to a vast range of opinions, considerable convergence exists on the need to develop an appropriate regulatory framework designed to govern germline editing ${ }^{[8]}$ In this regard, the release of the draft governance framework is noteworthy as it implies reception to the idea of germline editing. As such, in choosing the path of governance as opposed to prohibition, the WHO framework recommends a set of guidelines pertaining to germline editing to ensure that high ethical standards pave the way forward. The draft framework in part 2.1 provides that in permitting genome editing, there are two fundamental choices to be made. Firstly, consideration should be made of whether all potential applications of genome editing ought to be regulated by a single system or via a sector-specific approach. ${ }^{[2]}$ And secondly, a fundamental consideration remains on the appropriate degree of oversight. Where the potential risk of harm from the technology is low, then a far more permissive regulatory framework should be adopted. ${ }^{[2]}$

The draft framework in part 3.1 acknowledges heritable human genome editing (herein referred to as germline editing) as a special challenge. It acknowledges that'there will be significant differences in the policy directions taken by countries around the world regarding prohibition versus permission [...] and good governance must anticipate and plan for these variations. ${ }^{[2]}$ The above wording of the draft framework therefore seems to suggest a permissive approach toward germline editing, subject to proper regulation.

The draft framework nevertheless suggests that, where possible, people should make use of more acceptable technological alternatives to germline editing, such as pre-implantation genetic testing (PGT) and adoption. Beyond that, the draft framework also recommends that policy decisions must be made 'about how much weight should be given to the desire to have genetically related offspring and whether such risks are tolerable in light of the available technological and social alternatives. ${ }^{[2]}$ When analysed critically, the draft framework may be interpreted to indicate that there really is no basis for pursuing germline editing, as the genetic conditions for which germline editing is most often recommended can be prevented by other technological alternatives, such as PGT. However, although PGT may frequently give intended parents the opportunity to have a healthy offspring, it is not an effective strategy in all situations. ${ }^{[9]}$ In addition, various authors have argued that germline editing has moral benefits over PGT. ${ }^{[10]}$ PGT necessitates the 'setting aside' of embryos based on a single genetic variation. Intrinsically, the genetic trait makes all other characteristics of that (would-be) individual negligible. While certain traits are viewed as incompatible with life, such a perception cannot be equated with individuals who merely seek to modify specific genetic traits. Accordingly, PGT may be seen by some to be more morally challenging than germline editing, and this raises the question of why germline editing should be seen as necessarily more morally reprehensible than genetic technologies such as PGT.

In conclusion, although certain sections of the draft framework are problematic, its regulatory approach is not rigid, and is open to a variety of views and perspectives, and does well to remain open to the possibility that states may elect to regulate rather than ban germline genome editing. This reaffirms principle 1, and illustrates why SA should adopt a similarly flexible and permissive regulatory position, which is open to a multiplicity of views on questions such as the morality of modifying the human genome.

\section{Principle 2: Use the well-established standard of safety and efficacy}

$\mathrm{SA}$, like most states, has mechanisms in place to exercise oversight over new technologies that seek approval to be placed on the market. This function is primarily carried out by health research ethics committees, which must provide approval for all research on human participants in terms of section 73 of the NHA. ${ }^{[6]}$ If, as it has been suggested, ${ }^{[6]}$ clinical applications of heritable genome editing fall within the ambit of the Medicines and 
Related Substances Act No. 101 of 1965, ${ }^{[1]]}$ then these may only be placed on the market if they are proven to be both safe and efficacious, as per section $1 .^{[3]}$ The standard of safety and efficacy as precondition to clinical use is well established: therefore it is unsurprising that this is the standard set by the Medicines and Related Substances Act, and the standard that heritable genome editing technologies would have to meet. But does this benchmark measure up to global standards?

The draft framework ostensibly acknowledges safety and efficacy as baseline requirements for human genome interventions, and further, highlights that some of the fundamental decisions to be taken by states regarding the regulation of human genome editing turn on the issue of how we weight safety and efficacy. ${ }^{[2]}$ For instance, the draft framework alludes to the conflict between strict approaches to research oversight, which might create high barriers for establishing safety that slow down the development of science, and more liberal approaches of oversight, which prioritise the need to promote innovation, and thus avoid excessively high standards. Although the draft framework itself avoids any commitment to a particular perspective, in its discussion on heritable human genome editing it highlights the fact that one of the core questions to be considered by states in regulating this area is what capacity exists for long-term, possibly multigenerational, monitoring of the health and safety of offspring. ${ }^{[2]}$ Beyond this, the draft framework provides little guidance on under what circumstances heritable genome editing technologies might be regarded as 'safe enough'.

The potential need for SA law to provide for mechanisms to monitor the multigenerational impacts of heritable genome editing is something that has already been identified as a gap in our regulation. ${ }^{[4]}$ Such long-term monitoring programmes are not entirely unheard of in the healthcare space, and capacity for this would have to leverage off these existing systems - such as mechanisms for pharmacological vigilance. SA has such mechanisms in place, run by governments, academics and non-governmental organisations however, it is argued that there is a need for an overarching plan for national pharmacovigilance. ${ }^{[12]}$ Given the success of these models in key areas, similar mechanisms could potentially be adapted for heritable genome editing technologies.

\section{Principle 3: Non-therapeutic uses of germline gene editing may be permissible}

One widespread morally contentious concern about germline editing is its potential use for human enhancement. Although the HPCSA ethical guidelines are silent on non-therapeutic uses of germline editing, efforts have been made by the Academy of Science of $S A$, through the drafting of consensus reports, toward an attempt to build on the regulation of human germline genome editing in SA.

Correspondingly, the draft framework admits that genome editing for enhancement purposes is problematic, as allowing it would deepen social inequalities, and hence enjoins us to consider 'whether genome editing for enhancement purposes should be permitted! ${ }^{[2]}$ It has been extensively argued that the overzealous use of germline editing for non-therapeutic purposes could affect future generations by stripping away forms of human diversity, and perpetuate social harm. ${ }^{[5]}$ A question that begs to be addressed is whether all forms of enhancement are bad. Perhaps not!
We posit that although it is easy to identify severe cases of enhancement, the same cannot be said about borderline cases. Inevitably, these borderline cases leave us with complicated scenarios in which it is impossible to decide whether a given clinical application qualifies as a therapy or as an enhancement. Accordingly, the draft framework suggests that the permissibility of germline editing for enhancement purposes should be subject to inclusive and transparent societal debate. Remarkably, the draft framework also recognises that societal concerns can vary depending on the type of enhancement and the context in which it will be used: therefore, successful governance would have to be adequately flexible to test the proposed enhancements in multiple contexts. ${ }^{[2]}$

We argue that while there are varying views about the permissibility of non-therapeutic uses of germline editing, it is undeniable that the concern that only a select few and not the vast majority of humanity will have access to it is another pressing issue. Hence, as a plausible approach to this concern, we suggest, firstly, that we should distinguish moral concerns about non-therapeutic uses of germline editing itself from its dissemination. In this way, concerns regarding unequal access will not obliterate the actual discourse on non-therapeutic uses of germline editing, and could be remedied using guiding principle five below.

\section{Principle 4: Respect parents' reproductive autonomy}

The advancement of human rights and freedoms is one of the founding values of the SA Constitution. ${ }^{[13]}$ This value informs the generally permissive stance by SA statutes and courts on the regulation of reproductive technologies. ${ }^{[14]}$ Therefore, while there is no law explicitly providing for heritable genome editing per se, SA's condonation of other new reproductive technologies such as in vitro fertilisation, gamete donation and pre-implantation genetic screening (even without a medical indication) are all support for the argument that heritable genome editing, if proven to be safe and efficacious, would fall within the rights of prospective parents to make decisions concerning reproduction, as per section 12(2)(a) of the Constitution. ${ }^{[4]}$ This is something that is not unique to the SA context, as rights relating to reproduction are recognised in a number of international human rights instruments, reaffirming the need for state actors to respect the autonomy of parents in matters relating to reproduction. ${ }^{[14]}$

Regrettably, reproductive autonomy is an issue that receives little attention in the draft framework. The sole explicit reference to reproductive rights is in regard to how the reproductive autonomy of the genetically altered child may be curtailed if the intended edits go awry. ${ }^{[2]}$ Nevertheless, the framework does take an approach to the regulation of heritable genome editing that is commensurate with reproductive autonomy, insofar as it takes the position that individual states may elect to permit heritable genome editing, provided said states exercise due caution and mindfulness of the consequences of doing so. In other words, the recommendations of the draft framework align with the notion that liberal democracies such as SA may have good cause for permitting heritable genome editing, such as the need to show respect for the reproductive autonomy of prospective parents.

\section{Principle 5: Promote the achievement of equality of access}

Social justice and non-discrimination are governing principles of the draft framework. In ensuring equitable access to the potential 
benefits of germline editing, the draft framework calls for the development of appropriate and feasible medical interventions that cater for the widest possible diversity of the population. It also provides that germline editing if approved for research and clinical applications, raises concerns with regard to fairness, social justice and non-discrimination, as well as potential disregard for the individual dignity of persons with disabilities.[2] And so, as with many of the questions raised by germline editing, 'how can the possible negative social effects of germline editing be addressed, including the risk of exacerbating social inequalities, if the new technology is not made accessible on an equitable basis? ${ }^{[8]}$ This, furthermore, reinforces the validity of the proposal by Thaldar et $a l .{ }^{[4]}$ that regulation on heritable genome editing should seek to support mechanisms for promoting access to the technology.

It is important to note that although SA is well known for its progressive Constitution that guarantees the right to access to healthcare services, such access has historically been distorted in terms of disability, ethnicity, gender and a number of other factors. Accordingly, the mechanisms developed to provide equal access to healthcare services remain overwhelmingly prejudicial towards certain individuals. In the case that germline editing is eagerly awaited by some patients, the potential high cost of treatment may cause accessibility barriers to those who await it. The emergence of germline editing for disease treatment will therefore likely lead to a contentious affordability debate. As proposed by the draft framework, what efforts can SA put in place to ensure that germline editing does not exacerbate unjust discrimination, for instance, by making its end use products more accessible (affordable)?

In 2011, the SA government released a Green Paper (National Health Insurance (NHI) policy paper) proposing the establishment of an $\mathrm{NHI}$ scheme. ${ }^{[15]}$ The $\mathrm{NHI}$ is a health support initiative intended to pool funds together to provide access to healthcare for all South Africans, based on their health needs, irrespective of their socioeconomic status. However, while this is a step in the right direction, the NHI skeleton lacks flesh in terms of the issue of service coverage, and thus it is unclear whether clinical applications of germline editing will ever fall within the prescribed formulary in order to permit coverage under $\mathrm{NHI}$.

\section{Conclusion}

Given the immense possibilities raised by the most recent advances in human genome editing technology, there is an urgent need for effective regulation of its clinical applications on both the international and national planes. The WHO draft framework has attempted to provide skeletal guidance on whether germline editing should be allowed, and if so, under what circumstances. While the draft framework does not set out 'cast in stone' regulatory rules, the draft framework has established momentum for exploring different regulatory options, particularly for SA. What emerges from the forgoing analysis is that the five principles proposed for regulatory reform in SA align with the vision of the draft framework, and further justify why SA law should adhere to these principles. It is for these reasons that we believe that if SA, or any liberal democracy, uses these principles as a guide, they will create a regulatory environment that is in step with what is likely to be the future of global regulation of germline genome editing.

Acknowledgements. None.

Author contributions. Equal contributions.

Funding. National Research Foundation, grant 116275; University of KwaZulu-Natal, African Health Research Flagship Grant; University of the Free State.

\section{Conflicts of interest. None.}

1. National Academies of Sciences. Human genome editing: Science, Ethics, and Governance. Washington, DC: National Academies Press, 2017.

2. World Health Organization Advisory Committee on Developing Global Standards for Governance and Oversight of Human Genome Editing. Human genome editing: A draft framework for governance. Geneva: WHO, 2020. https://www. who.int/docs/default-source/ethics/governance-framework-for-humangenome-editing-2ndonlineconsult.pdf?ua=1 (accessed 13 August 2020).

3. Shozi B. A critical review of the ethical and legal issues in human germline gene editing: Considering human rights and a call for an African perspective. S Afr J Bioeth Law 2020;13(1):62-67. https://doi.org/10.7196\%2FSAJBL.2020. v13i1.00709

4. Thaldar DW, Botes M, Shozi B, Townsend B, Kinderlerer J. Human germline editing: Legal-ethical guidelines for South Africa. S Afr J Sci 2020;116(9/10).https://doi. org/10.17159/sajs.2020/6760

5. Gyngell C. Gene editing and the health of future generations. J R Soc Med 2017;110(7). https://doi.org/10.1177\%2F0141076817705616

6. South Africa. National Health Act No. 61 of 2003.

7. Health Professions Council of South Africa. Booklet 14: General ethical guidelines for biotechnology research in South Africa. Guidelines for good practice in the health care professions. Pretoria: HPCSA, 2014 https://www.hpcsa.co.za/ Uploads/editor/UserFiles/downloads/conduct_ethics/Booklet\%2014.pdf (accessed 12 September 2020).

8. Yotova R. Regulating genome editing under international human rights law. $\mathrm{Br}$ Inst Int Comp Law. 2020;69:653-684.

9. Ranisch R. Germline genome editing versus preimplantation genetic diagnosis: Is there a case in favour of germline interventions? Bioethics 2020;34(1):60-69. https://doi.org/10.1111/bioe.12635

10. Gyngell C, Bowman-Smart $H$, Savulescu J. Moral reasons to edit the human genome: Picking up from the Nuffield report. J Med Ethics 2019;45. https://doi. org/10.1136/medethics-2018-105084

11. South Africa. Medicines and Related Substances Act No. 101 of 1965.

12. Mehta U, Dheda $M$, Steel G, et al. Strengthening pharmacovigilance in South Africa. S Afr Med J 2013;104(2):104-106. https://doi.org/10.7196/samj.7517

13. Constitution of the Republic of South Africa, 1996.

14. Shozi B. Something old, something new: Applying reproductive rights to new reproductive technologies in South Africa. S Afr J Hum Rights 2020;36(1):1-24. https://doi.org/10.1080/02587203.2020.1776632

15. South Africa. National Health Insurance in South Africa Policy Paper 2011. http:// pmg-assets.s3-website-eu-west-1.amazonaws.com/docs/110812nhi_0.pdf (accessed 10 November 2019).

Accepted 19 November 2021. 